\title{
APEOW: A Personal Persuasive Avatar for Encouraging Breaks in Office Work
}

\author{
Przemysław Kucharski ${ }^{1}$, Piotr Luczak $^{1}$, Izabela Perenc ${ }^{1}$, Tomasz Jaworski ${ }^{1}$, Andrzej Romanowski ${ }^{1}$, \\ Mohammad Obaid ${ }^{2}$, Paweł W. Woźniak ${ }^{3}$ \\ ${ }^{1}$ Lodz University of Technology, Łódź, Poland \{pkucharski, pluczak, izarenc, tjawors, androm, \}@iis.p.lodz.pl \\ ${ }^{2}$ KUAR, Media and Visual Arts, Koç University, Istanbul, Turkey, mobaid@ku.edu.tr \\ ${ }^{3}$ Chalmers University of Technology, Gothenburg, Sweden, pawelw@chalmers.se
}

\begin{abstract}
Proper break taking during office work iss necessary to prevent musculoskeletal disorders and reduce the risk of heart disease. We present APOEW - an avatar for preventing continuous office work without taking breaks. APOEW is a system that uses a personalized robot avatar to encourage proper break behaviour during office work. The avatar signals the need for a break by stooping. The system was designed to be unobtrusive and blend well with the office environment. The avatars are customisable in order to enable users to design their work environment freely. We conducted a user study where we observed developers working in front of their computers next to the avatar. Preliminary results indicate it has no negative impact on the work environment and users are intrigued by the system. Moreover, a survey on attitude to our concept reveals interesting and positive feedback that will help to develop an APOEW system further.
\end{abstract}

\section{INTRODUCTION}

$\mathbf{T}$ HE SEDENTARY nature of office work is universally recognised as detrimental to health [4]. Employees are advised to take regular breaks as this results in benefits to cardiovascular health. Yet, workers often forget about the need for break for a variety of reasons. As digital artefacts have already pervaded the work environment and users often have multiple devices present on their desks, we propose introducing an additional object that would remind workers of taking regular breaks. APOEW (pronounced ape-oh) is a humanoid physical avatar robot that alters its posture to suggest a break is needed. We designed an avatar which can easily integrate with the work environment and attempt at persuading the user to take a break without disrupting the work. Being a humanoid robot, it visualises possible negative changes to the user's posture if a proper break regime is not followed. In the remainder of this paper, we present the past work that motivated the creation of APOEW and describe the design process of the avatar. We then provide details about the implementation of the system and report on a preliminary user study. This paper concludes with a discussion of future directions for the design of tools that support proper posture and break taking.

This work-in-progress paper contributes: (1) the design and implementation of a persuasive physical avatar aimed at promoting a proper break regime in office work and (2) initial design insights for future work on personal avatars in office spaces.

\section{RELATED WORK}

Personal avatars (both virtual and physical) have been used for a variety of purposes such as remote collaboration [10] or instant messaging [11]. Past research indicates that avatars can have a persuasive effect and it has been observed in virtual worlds [6]. Our work explores how physical avatars may influence users and promote a particular behaviour. We also build on the fuzzy avatar [3] concept (i.e. an avatar that does not provide direct messages, but merely offers hints) and thus explore ambiguity as a factor in persuasion.

Design interventions in workspaces have a long history in Human-Computer Interaction (HCI). Our work is particularly concerned with systems that attempt to change user behaviour in the work environment and measure office activity. Miro [1] informed office workers on the overall emotional climate in the building. The Clouds [12] attempted at persuading users to use the stairs instead of the lift. These works inspire our research as they prove that introducing new artefacts to office spaces can benefit the communities of office workers.

As we interpret APOEW as a system that addresses the domain of designing persuasive technology [2], our work is highly influenced by past achievements in this domain. Nakajima et al. [9] used persuasive technology successfully to promote healthy habits such as brushing teeth. Hong et al. [7] addressed posture issues while working with computers through a flower-shaped avatar, which provided real-time feedback. A similar approach can be found in [14], where an abstract shape of avatar is designed to reflect human behaviour. Morris et al. [8] investigated how proper break behaviour may be elicited by encouraging hands-free interactions during breaks. Haller et al. [5] used a sensor chair to provide posture feedback through digital and physical means. Also [13] addressed the problem of sedentary lifestyle, but with the use of an ambient display. APOEW is interestingly different from the above past work as it uses a partly ambiguous physical avatar and its feedback is based on cumulative data.

\section{DESIGN}

Our design work was influenced by personal avatars described in past work as well as previous deployments of interactive technologies in office spaces. We imagined a scenario where an office worker could place a humanoid robot on their desk. The avatar would be personalisable in order 
to make it blend well with the rest of the office space and add a personal touch to the desk. In spaces where desks are assigned dynamically, it could function as an easy way to make the space cosier. Continuous, accumulated avoiding of the necessary breaks would result in APOEW stooping. It would also use audio output in extreme cases when a break is really needed. While audio output is bound to cause a distraction, we reckoned that actively refusing to take break must be prevented using more direct means. This action would serve as a direct persuasive factor to suggest a break is much needed. Fig.1 presents the design of APOEW in the form of design sketches.

Our system also explores ambiguity as resource for design. We aimed for APOEW to provide persuasive cues that are not obvious and do not simply impose particular behaviours on the user. We decided to use a humanoid robot as we believed it would make the user reflect on the long-term effects of not taking regular breaks during office work. Customisable clothing would enable the user to make the desk space more personal. We aimed for making it possible for the user to have the avatar represent them. This would potentially trigger additional reflection potential. Furthermore, we enabled the user to notify the system of actually taking a break by pressing a button on the back of the robot. As the robot was designed to stand next to the computer screen, this would require some movement, perhaps prompting a break, even if the user intended to cheat the system. As our design goal was reflection and realising the importance of break taking, we opted for selfmonitoring instead of an elaborate activity sensing system.

\section{IMPLEMENTATION}

APOEW was implemented iteratively and two major research prototypes were studied so far. First one was prototyped with the Lego Mindstorms NXT robotics set. There were two robots programmed using Java for the initial user study of the system. This prototype employed a single NXT controller, 'intelligent bricks', based on 32-bit Atmel AT91SAM7S256 main microcontroller (with $256 \mathrm{~KB}$ flash memory and $64 \mathrm{~KB}$ of RAM). The robot was additionally equipped with a custom 'backpack' with the RaspberryPi and dedicated speakers, so that the robot could play sounds of higher quality than those provided by the NXT platform and produce synthesised speech. Three motors and two touch sensors were used to move the humanoid body, allow it to stoop and facilitate user input. Custom-made clothing was made for the robot using paper and cloth. Fig. 2 depicts the constructed and programmed humanoid robot next to a programmer at work.

A schedule for changing the avatars posture according to continuous work time was implemented. The robot would move slightly after 50 minutes without a break to signal that a work cycle was about to end. A more significant movement informed about a full cycle ending, after 55 minutes of work. After 65 minutes, the posture of the robot changed significantly and, after 75 minutes, sounds were produced. These effects were even stronger if the worker was skipping multiple breaks during a day.

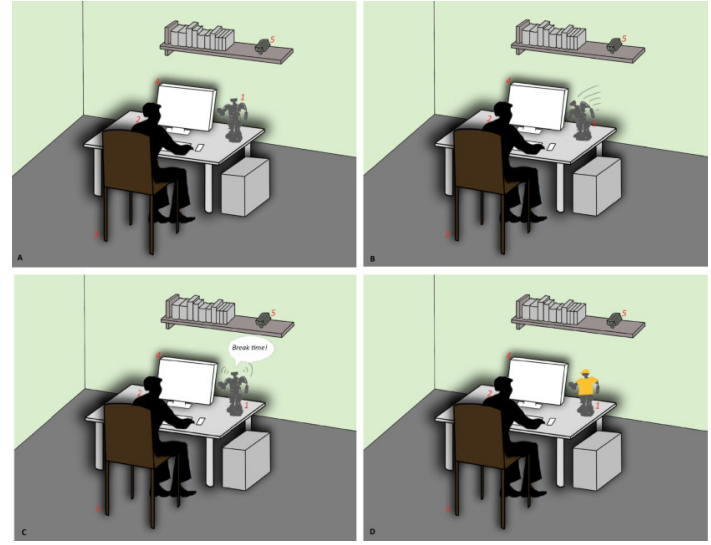

Fig. 1. Conceptual sketches for APOEW: (a) A personal avatar on an office desk. (b) APOEW suggests that a break is need by adjusting its posture. (c) A notification sound is played when the user excessively refuses to take a break. (d) A personalised version of APOEW featuring custom clothing.

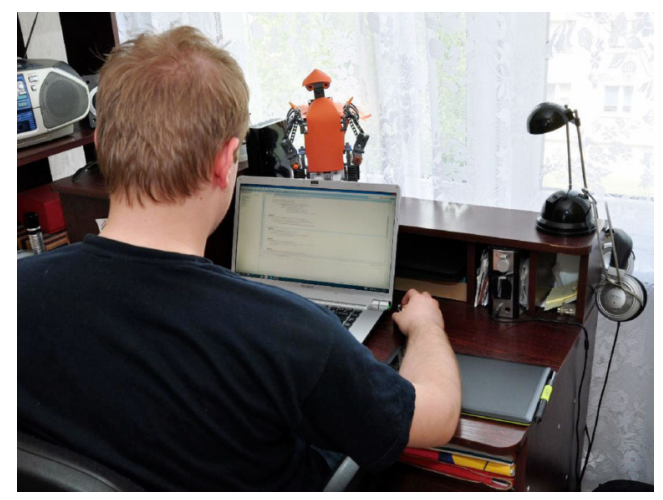

Fig. 2. The first implementation of APOEW. Note the custom clothing of the robot. APOEW is placed next to the computer screen so that interacting with the robot requires movement

\section{INITIAL EVALUATION}

We conducted a preliminary user study to assess the effectiveness of APOEW, verify the design assumptions and investigate the user experience created by the avatar.

APOEW was deployed in a controlled office environment. Four office workers (two developers and two graphic designers) from a local IT company were recruited for the study that lasted for three days. As work behaviour data was being gathered, a detailed privacy policy was presented and explained to the participants. We monitored two workers at a time using a video camera. A total of 96 hours of video was recorded ( 4 workers $\times 3$ days $\times 8$ hours). The first day of the study was used as a sample workday for comparison purposes. APOEW was introduced to the office space on day two. After the conclusion of the study, we performed semistructured interviews with the participants.

Three researchers performed independent qualitative analysis of the video and interview material. A joint discussion session was then held and observations were made. The introduction of the avatars to the office space drew attention 


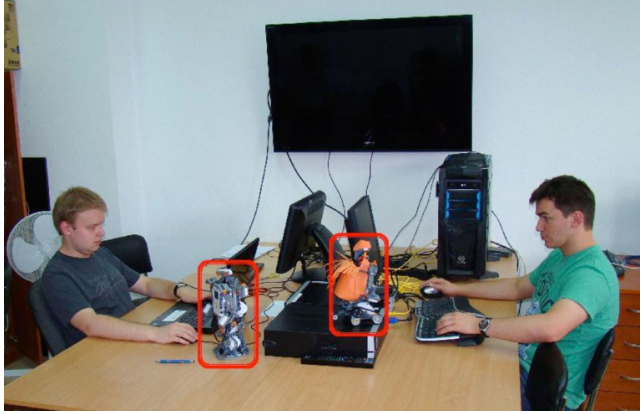

Fig. 3. An in-situ picture of the preliminary user study. Two IT professionals are performing their regular work with two APOEW (circled in red) units standing next to their screens. Four users spent a total of eight days working with APOEW.

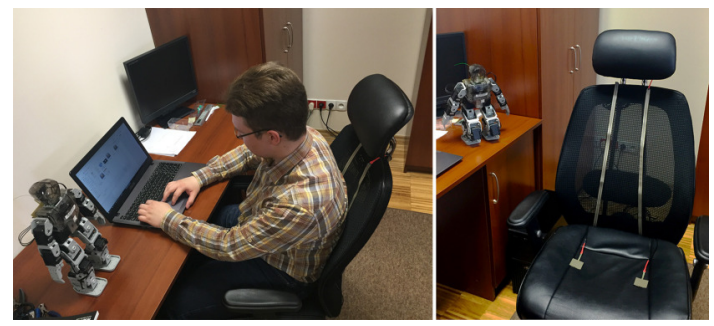

Fig. 4. The implementation of chair sensor during a second study - a Bioloid robot during the study on the left-hand side. Prototype sensors on the chair on the right-hand side.

of the participants. They were intrigued by APOEW and immediately started discussing its purpose. The participants quickly proceeded to personalize the robots on their desks, by adding features related to hobbies or favourite movies. The extra attention generated by APOEW suggests that our future work should concentrate on a long-term study of the system where the novelty effect of APOEW will be minimised. This is why this work-in-progress paper does not report quantitative data on the break taking behaviour of the participants. We also noted that the implementation of the prototype needs improvements - the noises generated by the servomotors may cause unintended distractions.

The next study was conducted using Bioloid robots and a prototype of a fully functional, yet not final, version of the system, i.e. we also prepared a set of sensors to be roughly mounted on top of chairs - on-top both of the seat and back of the chair. Such a design was to provide the automatic control of the system since the sensors were supposed to communicate the fact of the chair being released by the user and for how long, as well to trigger the APEOW procedure for persuasion if the chair is eventually not empty for a least 5 minutes. However, due to communication problems we were not able to conduct a proper study at that time. Fig. 4 shows the robot during the study with a Bioloid design (left-hand side of the picture) and a sensors prototype shown on the top: seat and back of an office chair. 2 Workers were monitored by 2 days each that gives in total 32 hours of this second design test.

In the interviews, users expressed their satisfaction at the

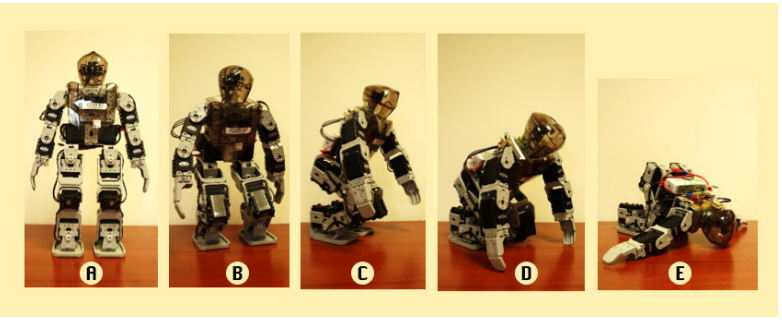

Fig. 5. The changes in the Avatar posture during the planned period.

fact the work environment became more attractive with the introduction of APOEW:

I really like this little fella. His bright clothing makes the office cosier.

The participants also wondered what would be the longterm effects of the system and suggested alternative uses or even peer pressure:

I'd like to see what happens when he stays here longer. I could also use this to see how in fact my friend is working or even pressure him to take a break through my avatar. And, I can dress him as Iron Man.

Overall, we can conclude that APOEW can potentially be integrated into an office environment and users are willing to consider the possibility of introducing personal avatars to their workspace. Privacy is a concern and we will aim to address that issue in future work. Further studies are required to measure the persuasive effect of APOEW.

In order to conduct further development of the project a survey was conducted to gain broader knowledge about the attitude towards the proposed concept. Fig. 5 shows the sequence of photos illustrating the consecutive phases of the APEOW persuasive movement.

$\mathrm{N}=198$ people responded to our survey including $41.4 \%$ women and $58.6 \%$ men. $47 \%$ working, $27.3 \%$ working and studying, $23.2 \%$ studying and $2.5 \%$ unemployed or retired. $47.2 \%$ of workers are employed in big companies related to IT business (1000+ employees). Results of the survey revealed that $59.6 \%$ declare that they do or try to do periodic breaks from sitting work while most of people (between $68.9 \%$ to $88.6 \%$ depending on the issue inquired) shown great concern about possible negative results of refraining from conducting these breaks. Feedback shown that majority of respondents express their positive attitude towards the concept as the following data show: Idea assessment: $58 \%$ positive vs. $21.2 \%$ negative and $20.8 \%$ neutral. Anticipated efficiency: $41.4 \%$ positive vs $26.8 \%$ negative and $31.8 \%$ neutral. Possible interfering with the work expected: $31.8 \%$ confirm vs. $43.4 \%$ not agree and $23.8 \%$ neutral. Expected deterioration of the work conducted: $16.6 \%$ confirm vs. $67.2 \%$ not agree and $16.2 \%$ neutral. As one can see from these numbers the APEOW concept was warmly welcomed by the respondents with more then half supporting the main idea and only nearly one fifth being explicitly against it. One third of users see potential interference with the work conducted positive generally but at 
the same time only about $17 \%$ foresee substantial disruption of the work and more than $67 \%$ not anticipating any significant issues with it. Another interesting results show possible ideas for how the proposed APEOW concept could work: Conviction of possibility to modify the look of the APEOW may strengthen its impact: Agree $36.4 \%$ vs $33.9 \%$ disagree and $29.7 \%$ not sure. Willing to personalize own APEOW: $60.6 \%$ by look: $38 \%$ using sound/voice: $27.2 \%$ adjusting the workbreak intervals: $77.8 \%$ other changes in persuasive behaviour procedure, etc.: $61 \%$. On the other hand, respondents think the following features would diminish the efficiency of the APEOW: personalisation: $11 \%$, sound/voice emission: $73.2 \%$.

Lastly, users answered questions related to anticipated adoption of the APEOW in the workplace and the results show that most of employers are expected to accept the proposed concept in their offices. 51\% respondents declared it explicitly, $29.3 \%$ said they would probably accept it vs. $5 \%$ disapprove at all and $13.1 \%$ rather disapprove.

\section{CONCLUSIONS AND FUTURE WORK}

This paper presented the design, implementation and initial evaluation of APOEW; a persuasive physical personal avatar that helps uses maintain a proper work break regime. The avatar was designed to integrate well with an office environment and offer personalisation features. We explored how a humanoid robot can trigger personal reflection in users. An initial user study with four participants working for three days showed that the robot gained that attention of the users and may have had impact on the workers work regime. The results also indicate that feedback forms require further investigation as privacy concerns were raised.

In the near future, we aim to improve the prototype by conducting further studies. We also aim to develop new interactions with the environment, e.g. the robot could make use of user's eye movements [15] or hand movements [16]. First, we aim to compare different types of posture, personalisation options and other output parameters and their effectiveness on altering break regimes. Next, we plan to run a longterm in-the-wild study that will verify the effectiveness of our approach.

In the larger picture, given the rapid advancement of robotics, we believe that HCI should address the role personal physical avatars can play in future everyday spaces. Our work explores only a fragment of what personal avatars may offer. We wonder how systems similar to APOEW can be used not only as persuasive technology, but also whether they can improve workflows, communication or social relationships. We hope that our work will inspire further inquiries into how physical avatars can be integrated in the computing landscape.

\section{ACKNOWLEDGMENTS}

Authors of this paper would like to express their deepest appreciation for Tomasz Kosiński, who has greatly contributed to the work on this project.

\section{REFERENCES}

[1] Kirsten Boehner, Rogerio DePaula, Paul Dourish, and Phoebe Sengers 2005. Affect: from information to interaction. Proceedings of the 4th decennial conference on Critical computing between sense and sensibility CC'05, ACM Press, 59. http://doi.org/10.1145/1094562.1094570

[2] Bj Fogg. 2009. A behavior model for persuasive design. Proceedings of the 4th International Conference on Persuasive Technology - Persuasive 09: 1. http://doi.org/10.1145/1541948.1541999

[3] William W. Gaver, Jacob Beaver, and Steve Benford. 2003. Ambiguity as a resource for design. Proceedings of the conference on Human factors in computing systems - CHI '03, ACM Press, 233. http://doi.org/10. $1145 / 642611.642653$

[4] Fred Gerr, Michele Marcus, and Carolyn Monteilh. 2004. Epidemiology of musculoskeletal disorders among computer users: Lesson learned from the role of posture and keyboard use. it Journal of Electromyography and Kinesiology 14: 25-31. http://doi.org/10.1016/j.jelekin.2003. 09.014

[5] Michael Haller, Christoph Richter, Peter Brandl, et al. 2011. Finding the right way for interrupting people improving their sitting posture. it Lecture Notes in Computer Science (including subseries Lecture Notes in Artificial Intelligence and Lecture Notes in Bioinformatics), 1-17. http://doi.org/10.1007/978-3-642-23771-3_1

[6] Austen L. Hayes, Amy C. Ulinski, and Larry F. Hodges. 2010. That avatar is looking at me! social inhibition in virtual worlds. it IVAâÁŹ10 Proceedings of the 10th international conference on Intelligent virtual agents, Springer-Verlag, 454-467.

[7] Jeong-ki Hong, Sunghyun Song, Jundong Cho, and Andrea Bianchi. 2015. Better Posture Awareness through Flower-Shaped Ambient Avatar. it Proceedings of the Ninth International Conference on Tangible, Embedded, and Embodied Interaction - TEI '14, ACM Press, 337-340. http://doi.org/10.1145/2677199.2680575

[8] Dan Morris, A.J. Bernheim Brush, and Brian R. Meyers. 2008. SuperBreak: using interactivity to enhance ergonomic typing breaks. it Proceeding of the twenty-sixth annual CHI conference on Human factors in computing systems - CHI '08, ACM Press, 1817. http://doi.org/10. 1145/1357054.1357337

[9] Tatsuo Nakajima, Vili Lehdonvirta, Eiji Tokunaga, and Hiroaki Kimura. 2008. Reflecting human behavior to motivate desirable lifestyle. it Proceedings of the 7th ACM conference on Designing interactive systems DIS '08, ACM Press, 405-414. http://doi.org/10.1145/1394445.1394489

[10] Oyewole Oyekoya, William Steptoe, and Anthony Steed. 2012. SphereAvatar: a situated display to represent a remote collaborator. it Proceedings of the 2012 ACM annual conference on Human Factors in Computing Systems - CHI '12, ACM Press, 2551. http://doi.org/10.1145/ 2207676.2208642

[11] Nadya Peek, David Pitman, and Richard The. 2009. Hangsters: tangible peripheral interactive avatars for instant messaging. it Proceedings of the 3rd International Conference on Tangible and Embedded Interaction - TEI '09, ACM Press, 25. http://doi.org/10.1145/1517664.1517675

[12] Yvonne Rogers, William R. Hazlewood, Paul Marshall, Nick Dalton, and Susanna Hertrich. 2010. Ambient influence: can twinkly lights lure and abstract representations trigger behavioral change? it Proceedings of the 12th ACM international conference on Ubiquitous computing Ubicomp '10, ACM Press, 261. http://doi.org/10.1145/1864349.1864372

[13] Regan Mandryk, Kathrin M. Gerling and Kevin G. Stanley. 2014. Designing Games to Discourage Sedentary Behaviour. it Playful User Interfaces: Interfaces that Invite Social and Physical Interaction, Springer Singapore, 253. http://dx.doi.org/10.1007/978-981-4560-96-2_12

[14] Nassim Jafarinaimi, Jodi Forlizzi, Amy Hurst, and John Zimmerman. 2005. Breakaway: An Ambient Display Designed to Change Human Behavior. it CHI 05 Extended Abstracts on Human Factors in Computing Systems, ACM Press, 1945. http://doi.acm.org/10.1145/1056808. 1057063

[15] Adam Wojciechowski and K. Fornalczyk. 2015. Single web camera robust interactive eye-gaze tracking method. Bulletin of the Polish Academy of Sciences Technical Sciences, 63, no. 4, pp. 879-886, 2015DOI: 10.1515/bpasts-2015-0100

[16] Mateusz Półrola and Adam Wojciechowski. 2012. Real-time hand pose estimation using classifiers. International Conference on Computer Vision and Graphics, Springer Berlin Heidelberg. 